\title{
Towards Tangible Interaction in Scraping Therapy
}

\author{
Casey Walker \\ Michael Jones \\ caseywalker117@gmail.com,m@byu.edu \\ Dept. of Computer Science \\ Brigham Young University \\ Provo, Utah
}

\author{
Steven Orrock \\ Karen Carter \\ Orrock/Mendenhall Sports Medicine and \\ Physical Therapy \\ Orem, Utah
}

\begin{abstract}
This work in progress explores tangible interaction in the context of scraping tools commonly used in physical Therapy. Physical therapy is a health profession which uses mechanical force or motion to improve a person's mobility and physical motion. We began with an open-ended research through design process that resulted in a scraping tool that gives the PT feedback on applied force using embedded pressure sensors and an ambient light display. At the conference, we will display a prototype interactive scraping tool and several other design artifacts. We invite participants to use the tool on a human calf simulant made from ballistics gel. We hope our work-in-progress will encourage new work exploring tangible interaction and physical therapy.
\end{abstract}

\section{CCS CONCEPTS}

- Human-centered computing $\rightarrow$ Human computer interaction (HCI).

Permission to make digital or hard copies of part or all of this work for personal or classroom use is granted without fee provided that copies are not made or distributed for profit or commercial advantage and that copies bear this notice and the full citation on the first page. Copyrights for third-party components of this work must be honored. For all other uses, contact the owner/author(s)

TEI '20, February 9-12, 2020, Sydney, NSW, Australia

(C) 2020 Copyright held by the owner/author(s).

ACM ISBN 978-1-4503-6107-1/20/02.

https://doi.org/10.1145/3374920.3374993 


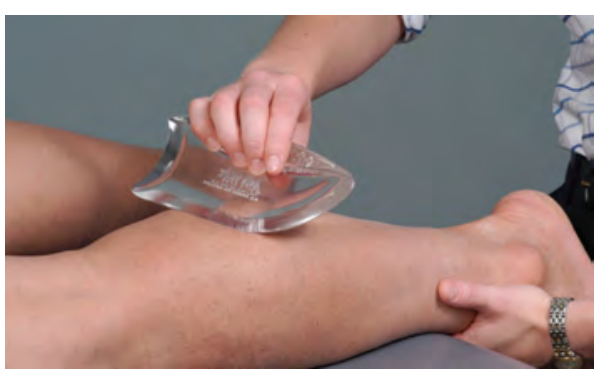

Figure 1: Scraping is a kind of soft tissue therapy intended to promote healing for soft tissue injuries.

${ }^{1}$ https://grastontechnique.com/

${ }^{2}$ fhttps://www.shape.com/lifestyle/mind-andbody/gua-sha-scraping-therapy-alleviatemuscle-pain

${ }^{3}$ https://www.astym.com/

\section{KEYWORDS}

scraping therapy, tangible interaction

\section{ACM Reference Format:}

Casey Walker, Michael Jones, Steven Orrock, and Karen Carter. 2020. Towards Tangible Interaction in Scraping Therapy. In Fourteenth International Conference on Tangible, Embedded, and Embodied Interaction (TEI '20), February 9-12, 2020, Sydney, NSW, Australia. ACM, New York, NY, USA, 6 pages. https://doi.org/10.1145/3374920.3374993

\section{INTRODUCTION}

Scraping is one form of hands-on care used in physical therapy. Scraping involves using a hand tool to scrape the skin that lies over scar tissue located in the superficial tissues that surround muscle, ligaments or tendons. Scar tissue in these superficial tissues can limit mobility. Figure 1 shows a scraping tool applied to a forearm. Scraping therapy triggers localized inflammation which in turn triggers a process that breaks down scar tissue and restores mobility.

The basic form of tools used for scraping have changed little over the centuries since the precursors of the method were developed [1,5]. Scraping tools vary by shape with different geometry designed to accommodate the contour of the body part being scraped. For example, each distinct edge of the tool shown in Figure 1 is designed to scrape a different region of the body. Several sets of scraping tools have been developed including Graston ${ }^{1}$, Gua Shua ${ }^{2}$ and ASTYM $^{3}$. Gua Shua being much older, with Graston and ASTYM being developed relatively recently. Although there are several tool sets that exist, these tools are static objects that contain no interactive computing.

In this work-in-progress, we seek to understand how interactive computing with tangible objects improves scraping therapy. Our methods are based on research through design (RtD) as it has been applied in human-computer interaction $[8,12]$. We chose RtD because it allows us to frame and re-frame the problem while work through design iterations in an underconstrained environment. In our work, an artifact is a prototype that embodies the insight gained in the project.

Our work is grounded in Toyama's assertion that interactive computing amplifies human capability and intent [11]. Grounding our work in Toyama's law of amplification respects the interaction between PT and patient by putting the actual technology in the background.

The participants in the project, who are the authors of this paper, are a design student with professional experience (including 2 products currently on the market), two PTs with 44 years of combined experience respectively and an experienced academic $\mathrm{HCl}$ researcher.

Over a 10 month period, our collaboration iterated on 7 distinct tool sets through 3 unique design phases. This process ultimately resulted in a scraping tool that uses light to give passive feedback on the amount of force applied during therapy. The design artifact embodies our new understanding of how tangible interaction could improve scraping therapy. 


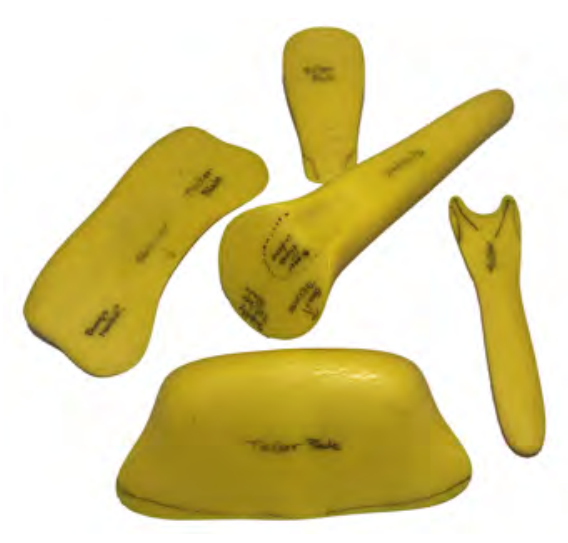

Figure 2: Annotations on 3D printed scraping tool prototypes facilitated communication between designers and PTs.
After reflecting on the process in its entirety, we propose that force is the dominant theme in the design of interactive tangible devices for scraping therapy. We mean "force" in the sense of physical force transmitted from the therapist to the patient through the scraping tool. In tool design, the transfer, measurement and communication of force impacts the shape, materials and electronics used in a scraping tool. Our artifact combines these elements in order to give the therapist objective feedback on how much force they are applying relative to a target force. Other combinations of these elements for other purposes may result in different tools that impact scraping therapy.

Our work so far has resulted in a series of prototypes that we will bring to the conference. The final prototype is an interactive scraping tool that provides feedback on the amount of force applied through the scraping tool. We will bring these prototypes along with a human calf simulant made from balistics gel and invite participates to try out the tools on the simulant. These prototypes are shown in the figures in this paper. The video that accompanies this paper shows the final interactive prototype in use. We hope our work will provoke discussions and future work on how tangible interaction can impact physical therapy for the human body.

\section{RELATED WORK}

While physical therapy has not been widely studied by interactive computing researchers, interactive computing has been studied by physical therapy researchers. Some of this work investigates the measurement of body position and motion. Examples include measuring the stability of the ankle joint [2], and measuring sitting position for patients with spinal cord injuries [7].

Rather than defining a problem and pursuing a solution, we consider an area of practice (scraping) and take a design-based approach to learning how tangible interaction might improve therapy. In this open-ended approach, we allow problems to emerge as we go and reflect on our experience. To our knowledge, our work is the first open-ended study of how interactive computing can improve physical therapy.

Our methods are based on research through design (RtD). Our approach to RtD is an iterative process in which designers frame and re-frame the problem as they iterate through artifacts. This is similar to Zimmerman et al.'s characterization of RtD which involves an "active process of ideating, iterating and critiquing potential solutions" [12]. In our work, the output of RtD is an artifact which embodies a set of ideas learned from the design process. During the process, we emphasize designing "the right thing" [3] rather than a viable product. Viable products satisfy constraints related manufacturing, consumer demand and cost which may not lead to useful basic research results.

Unlike Zimmerman et al., we do not frame scraping as a "wicked problem" (as originally described in [9]) which involves conflicting constraints. But we do frame tangible interaction in scraping as an "under-constrained problem" [12] which may benefit from a RtD approach. 


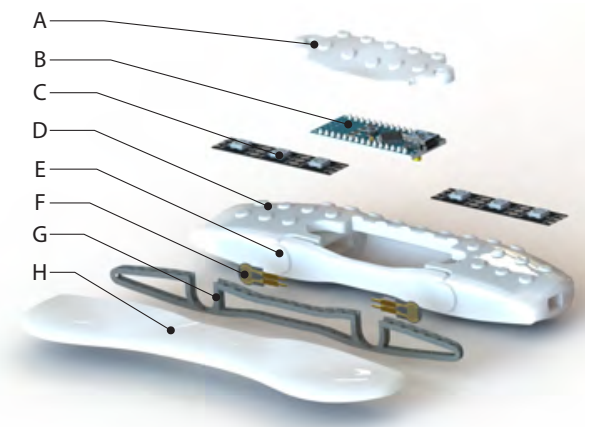

Figure 3: Exploded view of an interactive scrapping tool that provides feedback to the PT about the amount of force applied. Part $\mathrm{H}$ is the blade edge and part $\mathrm{F}$ is the force sensor embedded in the tool.

\section{TOOL SHAPE DESIGN}

We began with an open-ended observation of soft tissue scraping in physical therapy. Coming into this phase, we did not have a firm understanding of the questions that needed to be asked. With little to work with and no clear problem, we settled on an exploration phase to identify emergent issues. During a series of meetings over the course of 10 weeks, design efforts began to focus on the shape of the tool. We realized that the shape of the tool is important because it determines how force is generated by the PT and transmitted into the patient. Figure 2 shows a set of annotated scraping tools generated during one of these design sessions. Annotations on the 3D printed tools facilitated clear communication.

After a period of reflection, we realized that the shape of the tool's blade edge is a critical factor in the transmission of force. We then conducted a series of design sessions over an 8 week period to refine the shape of the tool edge. These sessions focused on the radius of the blade edge. It was vital that the blade not be too sharp but not too dull. A very sharp edge could penetrate the outer layers of skin, harming any potential patients. However a dull tool did not transmit force to the sub dermal tissue therefore removing the benefits of scraping therapy.

\section{TOOL INTERACTION DESIGN}

After designing tools shapes with the application of force as the key issue, we were surprised to find that there are no standardized means for quantifying the force or pressure applied during therapy. Force applied during scraping is often cast into three arbitrary categories: light, intermediate, and heavy. While some apply a numeric rating system between 1-10. Our tools from Phase's I and II are designed to carefully distribute force during therapy. But the amount of force applied by PTs is unregulated and not monitored.

The amount of force applied in scraping by different PTs has not been studied. But, the amount of force applied in other therapies has been studied and shows variation between therapists. For example, force applied by therapists in spinal mobilization includes "considerable variation" that differs by as much as $34 \%$ [4].

Based on that insight, we designed an interactive scraping tool that provides feedback to the PT about the amount of force applied. We decided to retrofit our tools to allow for embedded measurement of force. During therapy the tool would provide immediate feedback to the PT. Retrofitting a tool involved adding three embedded components to the tool: a force sensor embedded in a flexible gasket, a processor to read and monitor force values and output to give feedback.

The force sensor embedded in the tool needed to be small enough to fit into the tool without changing the shape of the tool. The sensor also needed to be accurate and able to measure force in the therapeutic range without clipping sensor readings. We selected the FSR 400 force sensing resistor 


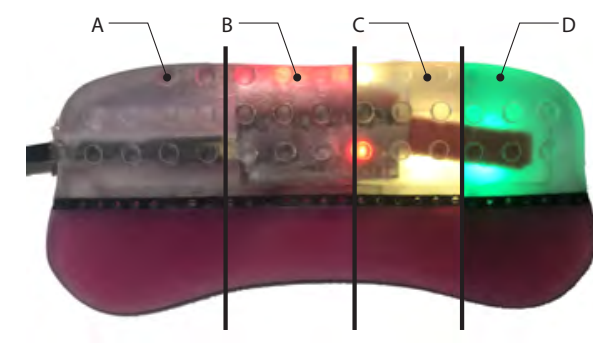

Figure 4: Composite image showing the four feedback states of the interactive scraping tool. A: Inert tool B: Red indication light indicates excessive force. $\mathrm{C}$ : Yellow light indicates insufficient force. $D$ : Green light indicates target force. We will bring this tool to the conference. due to its small diameter of $5.6 \mathrm{~mm}$, reliability, and range of $0.18-20.02 \mathrm{~N}$. A small micro controller was needed to control and read the sensors, the Arduino Nano was selected simply for the slim profile and wide range of capabilities.

The tool set was designed parametrically and then 3D printed this made them ideal for retrofitting them as smart tools. All components were modeled into Solidworks assuring they would fit into the model without altering the shape of the tool. Figure 3 shows the exploded components of the tool assembly that the houses sensors and the micro-controller. A gasket, component $\mathrm{G}$ in Figure 3, was designed to let the tool compress when in use, activating the force sensors. The gasket was placed close to the blade edge, component $\mathrm{H}$, without interfering with functionality. The gasket was modeled in place and printed in place using Tango Black photo polymer with a Shore value of 26-28 (A) and a compressive set of $4 \%-5 \%$. Contact pads, on component D, were designed to transfer the force into the senors. Multiple senors, labeled F, were arrayed equidistant from each edge to capture the distributed force. All sensors were then wired into the Arduino Nano micro-controller, component B, that resided inside the hilt, components $\mathrm{A}$ and $\mathrm{D}$, of the tool. A USB cable connecting the micro controller to the host PC was necessary as space was extremely limited in the hilt, off the shelf wireless modules simply did not fit.

We needed a method to give real time feedback for the PTs to see how much force was being applied. A simple method was to display the force as a real time graph on a laptop screen. A laptop with a data stream was setup next to PTs so they could get feedback as they used the tool. It was clear that the PTs were unable to focus on the therapy and the laptop screen at the same time. A new less distracting method was needed to convey applied force to the PTs.

We chose ambient color scattered from RGB LEDs placed in the tool to communicate feedback. Color is a simple means of conveying information, conveys enough information for our needs and does not interrupt therapy by making noise or vibrating. Our use of color as an notification is similar to the use of color to provide non-distracting notifications to rock climbers [6]. To convey colorized feedback several RGB LEDs, component C of Figure 3, were inserted into the hilt. The hilt was printed in a translucent photopolymer to allow light to be seen. This is similar to the use of light pipes in other 3D printed interactive objects [10].

A three value color scale consisting of red, yellow and green indicated whether or not the applied force was greater than, less than or within the target force range. Figure 4 is a composite image showing the different colors displayed in the tool. Part $A$ of the image shows the tool in the inert state with no color. The red in part $B$ indicates that too much force is applied, yellow in part $C$ indicates too little force and green in part D means that the force is within the target range. 


\section{CONCLUSION}

We hope that our work in progress encourages researchers and practitioners to study tangible interaction in the context of physical therapy. Interactive techniques may lead to better clinical outcomes for patients and a better therapy experience for PTs. In this work in progress, we have used an open-ended design-based process to identify a specific application area and to create a system for that application area. Ongoing work involves evaluating the user experience created by interactive scraping tools. Our work will focus on characterizing the performance of the scraping tool and exploring the interactive experience created for the PT.

\section{REFERENCES}

[1] Maximilian Braun, Miriam Schwickert, Arya Nielsen, Stefan Brunnhuber, Gustav Dobos, Frauke Musial, Rainer Lüdtke, and Andreas Michalsen. 2011. Effectiveness of Traditional Chinese "Gua Sha" Therapy in Patients with Chronic Neck Pain: A Randomized Controlled Trial. Pain Medicine 12, 3 (2011), 362-369. https://doi.org/10.1111/j.1526-4637.2011.01053.x

[2] Y.L. Chiu, Y.R. Hou, Y.J. Tsai, and W.H. Sung. 2018. Validity of smartphone-based postural control assessment for chronic ankle instability. Annals of Physical and Rehabilitation Medicine 61 (2018), e449. https://doi.org/10.1016/j.rehab.2018.05.1046 12th World Congress of the International Society of Physical and Rehabilitation Medicine. Paris. 8-12 July 2018.

[3] Christopher Frayling. 1993. Research in Art and Design. Royal College of Art Research Papers 1, 1 (1993), 1-9.

[4] M.c. Harms and D.I. Bader. 1997. Variability of forces applied by experienced therapists during spinal mobilization. Clinical Biomechanics 12, 6 (1997), 393-399. https://doi.org/10.1016/s0268-0033(97)00023-5

[5] Jooyoung Kim, Dong Jun Sung, and Joohyung Lee. 2017. Therapeutic effectiveness of instrument-assisted soft tissue mobilization for soft tissue injury: mechanisms and practical application. Journal of Exercise Rehabilitation 13, 1 (2017), 12-22. https://doi.org/10.12965/jer.1732824.412

[6] Felix Kosmalla, Frederik Wiehr, Florian Daiber, Antonio Krüger, and Markus Löchtefeld. 2016. ClimbAware: Investigating Perception and Acceptance of Wearables in Rock Climbing. In Proceedings of the 2016 CHI Conference on Human Factors in Computing Systems (CHI '16). ACM, New York, NY, USA, 1097-1108. https://doi.org/10.1145/2858036.2858562

[7] A.S. Perez-San Pablo, J. Quinzanos-Fresnedo, J.C. Lopez-Romero, and I. Quiñones-Uriostegui. 2018. Reliability of posturographic measures obtained during instrumental assessment of trunk control using inertial sensors in individuals with spinal cord injury. Annals of Physical and Rehabilitation Medicine 61 (2018), e84. https://doi.org/10.1016/j.rehab.2018.05.179 12th World Congress of the International Society of Physical and Rehabilitation Medicine. Paris. 8-12 July 2018.

[8] Majken Kirkegård Rasmussen, Timothy Merritt, Miguel Bruns Alonso, and Marianne Graves Petersen. 2016. Balancing User and System Control in Shape-Changing Interfaces: A Designerly Exploration. In Proceedings of the TEI '16: Tenth International Conference on Tangible, Embedded, and Embodied Interaction (TEI '16). ACM, New York, NY, USA, 202-210. https://doi.org/10.1145/2839462.2839499

[9] H.W.J. Rittel and M. M. Webber. 1973. Dilemmas in a general theory of planning. Policy Sciences (June 1973), 155-169.

[10] Valkyrie Savage, Ryan Schmidt, Tovi Grossman, George Fitzmaurice, and Björn Hartmann. 2014. A Series of Tubes: Adding Interactivity to 3D Prints Using Internal Pipes. In Proceedings of the 27th Annual ACM Symposium on User Interface Software and Technology (UIST '14). ACM, New York, NY, USA, 3-12. https://doi.org/10.1145/2642918.2647374

[11] Kentaro Toyama. 2010. Can technology end poverty? Boston Review 35, 5 (2010), 12-18,28-29.

[12] John Zimmerman, Jodi Forlizzi, and Shelley Evenson. 2007. Research Through Design As a Method for Interaction Design Research in HCl. In Proceedings of the SIGCHI Conference on Human Factors in Computing Systems (CHI '07). ACM, New York, NY, USA, 493-502. https://doi.org/10.1145/1240624.1240704 